\title{
Foreword of the Special Issue Dedicated to the 2013 Workshop on Approximation and Online Algorithms
}

\author{
Christos Kaklamanis • Kirk Pruhs
}

Published online: 8 April 2015

(C) Springer Science+Business Media New York 2015

\section{Introduction}

The eleventh Workshop on Approximation and Online Algorithms (WAOA) was part of the federated ALGO conferences, and was held September 5 and 6, 2013, in Sophia Antipolis, France. The field of approximation algorithms studies how closely we can approximate optimal solutions to problems that are known to be computationally hard to solve exactly. The field of online algorithms studies how closely we can approximate optimal solutions to problems where the algorithm must make decisions before all the necessary information is available. Although the difficulties arise from different sources, computational hardness vs. lack of information, these fields share many common techniques, most notably the use of worst-case relative error to measure the goodness of an algorithm. The purpose of this special issue is to provide a snapshot of the state of current research in approximation and online algorithms. The nine papers in this special issue were selected for invitation by the program committee of WAOA. The papers underwent the normal reviewing process for Theory of Computing Systems. We now briefly introduce and describe the approximation and online papers separately.

C. Kaklamanis

Department of Computer Engineering and Informatics, University of Patras and CTI "Diophantus",

Patras, Greece

e-mail:kakl@ceid.upatras.gr; ckakl@cti.gr

K. Pruhs $(\bowtie)$

Computer Science Department, University of Pittsburgh, Pittsburgh, US

e-mail: kirk@cs.pitt.edu 


\section{Approximation Algorithms}

The paper “On Fixed Cost k-Flow Problems” by Mohammad Taghi Hajiaghayi, Rohit Khandekar, Guy Kortsarz, and Zeev Nutov considers the setting of a network where there are operational costs on the links. The paper considers problems of choosing a low cost subnetwork that is sufficiently well-connected. The paper gives algorithms where the worst-case relative error only degrades slowly as the network grows.

The paper "Improved Approximation Algorithm for k-level Uncapacitated Facility Location Problem (with Penalties)" by Jaroslaw Byrka, Shanfei Li, and Bartosz Rybicki considers a problem involving determining where to open facilities of various types so as to minimize the cost of opening the facilities plus the cost of serving the customers, each of whom must be connected to one facility of each type. The paper gives an algorithm with bounded worst-case relative error.

The paper "Approximately counting approximately-shortest paths in directed acyclic graphs" by Matus Mihalak, Rastislav Sramek, and Peter Widmayer considers the problem of counting the number of paths from a specified source to a specified sink in a directed acyclic graph. The paper gives an algorithm that achieves arbitrarily good worst-case relative error.

The paper "Degree-Constrained Graph Orientation: Maximum Satisfaction and Minimum Violation" by Yuichi Asahiro, Jesper Jansson, Eiji Miyano, and Hirotaka Ono considers problems involving orienting edges in a graph so as to balance in the in-degree and out-degree of the nodes. The paper provides various results on how well some natural variations can be approximated.

The paper "Min-Sum 2-Paths Problems" by Trevor Fenner, Oded Lachish, and Alexandru Popa also considers problems involving orienting edges in a graph, here with the objective to minimize the aggregate length of disjoint paths between two designated pairs of vertices. The paper gives an algorithm that achieves arbitrarily good worst-case relative error.

The paper "Approximating the Sparsest k-Subgraph in Chordal Graphs" by Remi Watrigant, Marin Bougeret, and Rodolphe Giroudeau considers a problem that involves finding the sparsest part of a graph. The paper gives algorithms with good worst-case relative error on some commonly-arising special graph classes.

The paper "Low Dimensional Embeddings of Doubling Metrics" by Ofer Neiman considers problems involving low-distortion embedding more complex metric spaces into less complex metric spaces. The paper shows that this is possible for various metric spaces of special interest.

\section{Online Algorithms}

The paper "Online Knapsack Revisited” by Marek Cygan, Lukasz Jez, and Jiri Sgall considers the situation where objects with sizes and values must be packed into bins as they arrive over time. The main contribution of the paper is to determine under which objectives bounded worst-case relative error can be achieved.

The paper "On two continuum armed bandit problems in high dimensions" by Hemant Tyagi, Sebastian Stich, and Bernd Gartner considers the classic multi-armed 
bandit problem, in which at each time step one must choose from some collection of possible strategies. The goal is to choose strategies in such a way that the aggregate profit that one achieves is comparable to the best profit one could obtain from sticking to a single strategy. This paper shows that if the way in which profits are determined is in some sense sufficiently simpler than the choice of strategies, then this goal is achievable. 